\title{
Unique behavior of brain metastases during the treatment of nivolumab for metastatic renal cell carcinoma
}

\author{
Tsunenori Kondo $^{1,2}$, Hiroki Ishihara ${ }^{2}$ \\ ${ }^{1}$ Department of Urology, Tokyo Women's Medical University Medical Center East, Tokyo, Japan; ${ }^{2}$ Department of Urology, Tokyo Women's Medical \\ University, Tokyo, Japan \\ Correspondence to: Tsunenori Kondo, MD, PhD. Department of Urology, Tokyo Women's Medical University Medical Center East, 2-1-10, Nishiogu, \\ Arakawa-ku, Tokyo 116-8567, Japan. Email: tkondo@twmu.ac.jp. \\ Provenance: This is an invited article commissioned by the Section Editor Dr. Xiao Li (Department of Urology, Jiangsu Cancer Hospital \& Jiangsu \\ Institute of Cancer Research \& Affiliated Cancer Hospital of Nanjing Medical University, Nanjing, China). \\ Comment on: Flippot R, Dalban C, Laguerre B, et al. Safety and Efficacy of Nivolumab in Brain Metastases From Renal Cell Carcinoma: Results of \\ the GETUG-AFU 26 NIVOREN Multicenter Phase II Study. J Clin Oncol 2019;37:2008-16.
}

Submitted Oct 02, 2019. Accepted for publication Dec 02, 2019.

doi: $10.21037 /$ atm.2019.12.46

View this article at: http://dx.doi.org/10.21037/atm.2019.12.46

Brain metastases (BM) is one of the most unfavorable metastatic outcomes occurring in patients with renal cell carcinoma, and the overall survival (OS) of the patients with $\mathrm{BM}$ is approximately 1 year even after the advent of targeted therapy $(1,2)$. BM may also cause neurological symptoms, which significantly reduces the patients' quality of life. Thus, the control of BM is very important for the management of patients with metastatic renal cell carcinoma (mRCC). Nivolumab, an anti-PD-1 monoclonal antibody, has been shown to be effective in prolonging the survival of mRCC patients in the $2^{\text {nd }}$ or later line settings according to Checkmate025 (3). However, patients with BM had been excluded from this randomized trial, and thus, the efficacy of nivolumab in patients with BM remains undetermined.

A group of French physicians conducted a multicenter phase II prospective study (GETUG-AFU 26 NIVOREN: NCT03013335) where they administered nivolumab into patients along with at least 1 anti-angiogenic treatment in a setting that was similar to the real-world practice (4). They also included patients with BM in this study, and reported the safety and efficacy of nivolumab in a relatively large number of patients (5). In my opinion, they have shown some interesting points in the management of BM during the nivolumab therapy. I found that there are 4 specific points in this study that are significant. First, nivolumab is likely to prolong the OS of the patients with BM to 14 to 18 months, which might be slightly longer than the OS of the patients who have undergone targeted therapy.
Second, nivolumab activity is limited in BM compared to extracranial lesions. Third, the responses observed in the intracranial and extracranial lesions can be conflicting. Finally, administration of nivolumab to the patients with untreated BM did not cause symptomatic neurological adverse events, which lowers the chances of the treatment being discontinued.

First of all, this study shows that the OS of the patients with BM was 14-18 months despite concomitant radiotherapy (5). It was noted that although $12 \%$ of the patients showed complete remission after treatment with nivolumab alone, all the tumors that responded to the treatment were small in size $(<1 \mathrm{~cm})$. As I mentioned above, the OS of the patients with BM remain poor at approximately 1 year even after targeted therapy $(1,2)$. Majority of these patients had undergone some form of radiotherapy. Thus, nivolumab alone may slightly prolong the OS of patients with BM compared to those treated with anti-angiogenic therapy. Moreover, the treatment strategy for RCC is recently shifting to combination therapy. A combination of nivolumab and ipilimumab, or pembrolizumab and axitinib, has become new standard of treatment as the $1^{\text {st }}$-line therapy for RCC (6). Although the benefit of this combination therapy has not been clarified yet for patients with BM, these new treatment approaches are promising.

The second point this study showed is that the response observed in the intracranial lesions after treatment with 
nivolumab alone is lower than the response seen in extracranial ones (5). The objective response rate of the intracranial lesions is $12 \%$, which is lower than that of the extracranial lesions (21\%). Thus, it is likely that nivolumab alone is not adequate enough to treat BM. The authors compared the intracranial progression-free survival in patients with $\mathrm{BM}$ that were treated with radiotherapy to the untreated ones, and showed that it was shorter in the untreated patients (4.8 versus 2.7 months). Stereotactic radiosurgery (SRS) has been reported to be effective in prolonging the survival and controlling the symptoms of the patients (7). As the morbidity associated with SRS is very low, the patients with BM may benefit from SRS before starting systemic therapy. While this treatment strategy has been employed since the cytokine era, we should retain the same principle even in the era of immune checkpoint inhibitors (ICIs). In addition, screening for the presence of metastasis in the central nervous system before starting the nivolumab therapy is important. It still remains unclear whether the combination of nivolumab and ipilimumab, which is a current standard therapeutic approach (8), is also more effective against BM in RCC. A recent phase II study on metastatic melanoma has shown that this combination therapy elicited significant intracranial response in untreated $\mathrm{BM}$ in melanoma patients with the objective response at $57 \%$ (9). Further studies will let us know whether we can utilize SRS in the new era.

The third point is that intracranial response does not always correspond with that of extracranial lesions. In this study, 6 patients out of $34(18 \%)$ shows conflicting responses between brain and body assessment (5). Out of the 7 patients who showed partial response in the extracranial lesions, 3 patients displayed stable or progressive disease in $\mathrm{BM}$ as the best response. This indicates that new sites of BM might have developed in these patients in spite of well-controlled extracranial lesions. In fact, Zahoor et al. examined 23 patients who developed progressive disease with new organ sites of metastases during nivolumab therapy (10). Of these, 8 patients (35\%) showed new metastases in the brain, which is the most common site. We recently reported relatively high incidence of newly developed BM ( 6 out of $21,28.6 \%$ ) during nivolumab therapy in RCC (11). Out of 6 patients who developed new lesions in the brain, 4 of them had sustained objective response at the extracranial lesions. These contradictory responses have been rarely observed in the targeted era. It still remains unclear whether these discrepancies are limited only to ICIs. A possible explanation for this might be the poor penetration of the monoclonal antibodies across the blood brain barrier. However, studies have shown that nivolumab can penetrate well into the cerebrospinal fluid (12), and thus, it is hard to fully explain the reason behind this. Nivolumab alone has been used for patients who failed to respond to previous treatments due to which the tumors had become more aggressive and heterogenic before treatment with nivolumab. This might be the reason behind the conflicting responses.

Finally, the fourth point is that nivolumab administration in patients with untreated BM was found to be safe, which reduced the chances of discontinuation of treatment. We are aware of the higher risk of intracerebral hemorrhage in the patients with BM treated with agents targeting vascular endothelial growth factor (VEGF) receptors (13). This might be attributed to development of hypertension or disturbances in the endothelial cell-platelet interaction caused by VEGF blockage. As the anti-tumor activity of ICIs is not directly associated with angiogenesis or coagulation, administering nivolumab upfront should be theoretically safe in patients with $\mathrm{BM}$ who are untreated. However, we have reported a patient who developed cerebral hemorrhage from BM just after initiation of nivolumab therapy (14). We suspect that this might be due to the accelerated progression of $\mathrm{BM}$, and this adverse effect has also been reported by other investigators $(15,16)$. In our experience with nivolumab treatment, this event did not occur when the patients with BM were subjected to radiotherapy prior to ICI therapy. Although these neurological adverse events may be infrequent, we should be aware of this possibility before treatment with ICIs is initiated.

As we have discussed, the GETUG-AFU 26 NIVOREN brain metastasis study clearly provides substantial information on the treatment of mRCC patients with BM with nivolumab. This study has shown that nivolumab might improve the survival of the RCC patients with BM. Imaging should be performed to screen and examine the status of brain metastasis before starting nivolumab treatment. Additionally, the lesions, if found, should be treated with radiotherapy including SRS first, and then with nivolumab. Although its influence on the OS of patients remains undetermined, radiotherapy provides better local control of the metastasis in the brain. Further investigations are warranted to clarify if this strategy can be applied in the future.

\section{Acknowledgments}

The authors thank Ms Nobuko Hata (Department 
of Urology, Tokyo Women's Medical University) for secretarial work.

\section{Footnote}

Conflicts of Interest: Tsunenori Kondo received honoraria from Pfizer, Novartis, and Ono Pharmaceutical. H Ishihara has no conflicts of interest to declare.

Ethical Statement: The authors are accountable for all aspects of the work in ensuring that questions related to the accuracy or integrity of any part of the work are appropriately investigated and resolved.

\section{References}

1. Yildiz I, Bilici A, Karadurmuş N, et al. Prognostic factors for survival in metastatic renal cell carcinoma patients with brain metastases receiving targeted therapy. Tumori 2018;104:444-50.

2. Suarez-Sarmiento A Jr, Nguyen KA, Syed JS, et al. Brain Metastasis From Renal-Cell Carcinoma: An Institutional Study. Clin Genitourin Cancer 2019;17:e1163-70.

3. Motzer RJ, Escudier B, McDermott DF, et al. Nivolumab versus Everolimus in Advanced Renal-Cell Carcinoma. N Engl J Med 2015;373:1803-13.

4. Escudier B, Albiges L. Nivolumab in Patients With Metastatic Renal Cell Carcinoma Who Have Progresses During or After Prior Systemic Anti-angiogenic Regimen (NIVOREN). 2017. Available online: https://clinicaltrials. gov/ct2/show/NCT03013335.

5. Flippot R, Dalban C, Laguerre B, et al. Safety and Efficacy of Nivolumab in Brain Metastases From Renal Cell Carcinoma: Results of the GETUG-AFU 26 NIVOREN Multicenter Phase II Study. J Clin Oncol 2019;37:2008-16.

6. Ljungberg B, Albiges L, Abu-Ghanem Y, et al. European Association of Urology Guidelines on Renal Cell Carcinoma: The 2019 Update. Eur Urol 2019;75:799-810.
7. Haque $W$, Verma V, Butler EB, et al. Utilization of Stereotactic Radiosurgery for Renal Cell Carcinoma Brain Metastases. Clin Genitourin Cancer 2018;16:e935-43.

8. Motzer RJ, Tannir NM, McDermott DF, et al. Nivolumab plus Ipilimumab versus Sunitinib in Advanced Renal-Cell Carcinoma. N Engl J Med 2018;378:1277-90.

9. Tawbi HA, Forsyth PA, Algazi A, et al. Combined Nivolumab and Ipilimumab in Melanoma Metastatic to the Brain. N Engl J Med 2018;379:722-30.

10. Zahoor H, Barata PC, Jia X, et al. Patterns, predictors and subsequent outcomes of disease progression in metastatic renal cell carcinoma patients treated with nivolumab. J Immunother Cancer 2018;6:107.

11. Ishihara $\mathrm{H}$, Kondo $\mathrm{T}$, Takagi $\mathrm{T}$, et al. Newly developing brain metastases during nivolumab therapy for metastatic renal cell carcinoma: A case series in two Japanese institutions. Int J Urol 2019;26:849-50.

12. van Bussel MTJ, Beijnen JH, Brandsma D. Intracranial antitumor responses of nivolumab and ipilimumab: a pharmacodynamic and pharmacokinetic perspective, a scoping systematic review. BMC Cancer 2019;19:519.

13. Pouessel D, Culine S. High frequency of intracerebral hemorrhage in metastatic renal carcinoma patients with brain metastases treated with tyrosine kinase inhibitors targeting the vascular endothelial growth factor receptor. Eur Urol 2008;53:376-81.

14. Ishihara H, Kondo T, Takagi $\mathrm{T}$, et al. Immediate Progressive Disease in Patients with Metastatic Renal Cell Carcinoma Treated with Nivolumab: a Multi-Institution Retrospective Study. Target Oncol 2018;13:611-9.

15. Champiat S, Dercle L, Ammari S, et al. Hyperprogressive Disease Is a New Pattern of Progression in Cancer Patients Treated by Anti-PD-1/PD-L1. Clin Cancer Res 2017;23:1920-8.

16. Kato S, Goodman A, Walavalkar V, et al. Hyperprogressors after Immunotherapy: Analysis of Genomic Alterations Associated with Accelerated Growth Rate. Clin Cancer Res 2017;23:4242-50.
Cite this article as: Kondo T, Ishihara $\mathrm{H}$. Unique behavior of brain metastases during the treatment of nivolumab for metastatic renal cell carcinoma. Ann Transl Med 2019;7(Suppl 8):S267. doi: 10.21037/atm.2019.12.46 